\title{
COMPRENDRE la science du lidar
}

\author{
Pierre H. FLAMANT \\ LATMOS Laboratoire Atmosphère \\ Milieux Observations Spatiales, \\ Sorbonne Université, \\ 4 place Jussieu, \\ 75252 Paris Cedex 05, France \\ pierre.flamant@latmos.ipsl.fr
}

In memoriam Gérard Mégie apport du LIDAR aux sciences atmosphériques et géophysiques a été démontré sitôt l'avènement du laser par l'observation des nuages et des aérosols et la mesure de la distance Terre-Lune. La décennie 1970 a été consacrée à la mise en forme des méthodes. Les applications purement télémétriques n'ont commencé qu'en 1990. Laser et LIDAR sont indissociables, les progrès de l'un entraînant l'avancée de l'autre. Au cours des décennies 2000-2020, les formidables progrès techniques dans tous les domaines clés: lasers, détecteurs et informatique ont permis la multiplication des applications géophysiques et industrielles. Les LIDARs topographiques ont été les premiers dans l'espace au début des années 1970. Ils étaient installés sur les Lunar Orbiters des missions NASA Apollo 15, 16 et 17. Vingt ans plus tard, LITE a été le premier LIDAR atmosphérique de la NASA dans l'espace.

\section{Communautés LIDAR}

Les LIDARs sont principalement utilisés dans deux domaines d'application : (1) atmosphère, et (2) topographie des surfaces naturelles ou bâties et bathymétrie. Les applications atmosphériques sont le fait d'une communauté scientifique internationale tournée vers la recherche et les services (voir le site «International Coordination Group for laser Atmospheric Studies » ou ICLAS) tandis que les applications topographiques font l'objet d'activités commerciales entre donneurs d'ordre et prestataires de service (voir le site «International Lidar Mapping Forum»).

\section{Le LIDAR (light detection and ranging) est une méthode de télédétection} optique active. Des lasers de plus en plus performants ont conduit à la formidable expansion des applications LIDAR dans les domaines géophysiques et industriels. On utilise des lasers impulsionnels pour la mesure de distance (télémétrie) à laquelle s'ajoutent des mesures de composition atmosphérique ou marine, de vitesse et de réflectivité des cibles (terrain, végétation, bâti urbain).

À présent, la communauté LIDAR atmosphérique se compose : (1) de groupes de recherche pour des applications nouvelles, ils développent instruments et logiciels, (2) de groupes de recherche appliquée utilisant des LIDARs commerciaux souvent mis en réseau, et (3) des utilisateurs de données LIDAR en accès libre.

La communauté télémétrique pour la topographie et la bathymétrie s'est tout de suite insérée dans une logique industrielle. Elle est présente à l'internationale. Des start-up traitent les problèmes de bout en bout. Elles utilisent le même équipement laser/ LIDAR pour différentes applications. Les fournisseurs de données sont équipés de LIDARs et de logiciels de traitement très performants pour produire des cartes topographiques et bathymétriques à partir des nuages de points. Les demandeurs de prestation n'interviennent pas sur le processus de fabrication des données.

\section{Méthodes LIDAR Actif et passif}

Le LIDAR est une méthode d'analyse optique dite active car elle utilise sa propre source d'énergie lumineuse LASER pour éclairer une zone d'atmosphère ou les cibles à étudier. La mesure LIDAR est non intrusive. La méthode LIDAR se distingue des méthodes radiométriques passives qui utilisent la lumière solaire ou le rayonnement thermique infrarouge émis par les objets. L'exemple de la photographie ordinaire avec ou sans flash de lumière illustre cette distinction. La source de lumière additionnelle permet des mesures en l'absence de lumière solaire.

\section{Concept LIDAR}

Le concept LIDAR associe:(1) un instrumentéquipéd'un ou plusieurs lasers, (2) des processus d'interaction lumière-matière avec des cibles microscopiques (molécules, particules en suspension dans l'air ou dans l'eau) ou macroscopiques (terrain, végétation, bâtiments, véhicules), (3) une propagation aller-retour du rayonnement laser puis de la lumière diffusée/réfléchie. Pour l'air, l'atténuation est liée à une faible visibilité due à la pollution et aux conditions météorologiques. Pour les milieux aquatiques elle est due à la turbidité.

Les applications reposent sur la télémétrie (mesure de distance) et la restitution des propriétés optiques du milieu et des cibles. Pour l'atmosphère et la bathymétrie ce sont les phénomènes de diffusion et d'extinction qui permettent de restituer les propriétés optiques des particules en suspension dans l'air ou l'eau et les concentrations en composés minoritaires. Comme pour toute méthode optique, les nuages épais de la basse atmosphère gênent voire rendent inopérantes les mesures LIDARs.

Pour les applications topographiques, c'est le nombre de points d'impact au mètre carré qui sert à établir les modèles numériques de terrain (MNT ou DEM pour digital elevation model) ou les modèles numériques d'élévation (MNE) pour la végétation et les bâtiments. Un système de balayage de la ligne de visée permet de décrire une nappe de points. Les LIDARs topographiques permettent « d'effacer» 
la végétation. Ils ne conservent que les surfaces nues. La technique est utilisée en archéologie pour restituer des vestiges d'occupation de territoire ou pour détecter des failles sismiques ou des impacts de météorites.

PourdénommeruninstrumentLIDAR atmosphérique il est d'usage d'associer le processus d'interaction lumière-matière, l'objectif de la mesure et/ou la zone d'altitude et d'y ajouter des informations comme micro-, mini-etc. Pour les LIDARs topographiques ou bathymétriques, c'est plus simple car l'objectif est la mesure précise de distance répétée un grand nombre de fois par seconde.

\section{Instrument LIDAR et mesures}

Un instrument LIDAR est composé d'un ou plusieurs lasers émettant des impulsions brèves, d'un télescope, d'un ensemble de détection comprenant des filtres optiques, des polariseurs et des détecteurs rapides, d'un convertisseur analogique-numérique, d'une informatique pour gérer le fonctionnement de l'instrument et enregistrer les signaux numériques et afficher les mesures en temps réel (figure 1). Toutes les analyses postérieures sont effectuées avec les signaux numériques : soustraction des bruits parasites, méthodes d'inversion pour obtenir les grandeurs recherchées.

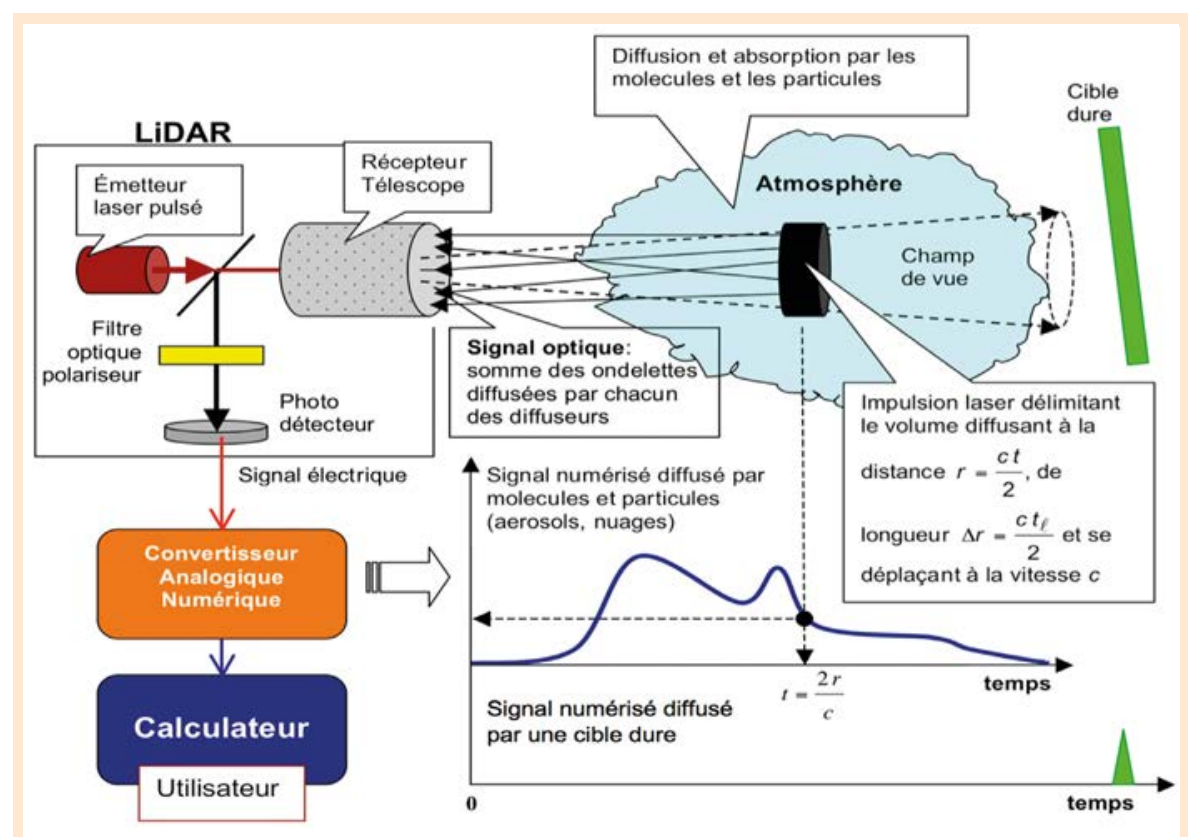

Figure 1. Mesures LIDAR atmosphérique et topographique : bloc instrument, interaction avec l'atmosphère, exemples de signaux numériques pour l'atmosphère (en bleu) et une cible dure (en vert).

Aujourd'hui, les lasers du commerce sont compacts, fiables et robustes. Différents types de lasers sont utilisés pour les applications LIDARs. On peut souligner l'omniprésence des lasers $\mathrm{Nd}^{3+}$-YAG qui émettent à $1,064 \mu \mathrm{m}$ et ce d'autant plus que les techniques de conversion en optique non linéaire permettent d'obtenir des longueurs d'onde plus courtes ou plus grandes. Il existe bien sûr d'autres catégories de lasers solides, fibrés ou à colorant qui offrent un très large choix de longueurs d'onde et de fréquence de répétition (nombre d'impulsions émises par seconde).

Par exemple, les applications télémétriques utilisent des lasers $\mathrm{Nd}^{3+}$ YAG avec des fréquences de répétition de $10^{3}$ à $10^{5} \mathrm{~Hz}$.

Il existe quatre grandes catégories d'instruments pour les applications atmosphériques suivant les processus d'interaction mis en jeux : (1) LIDAR

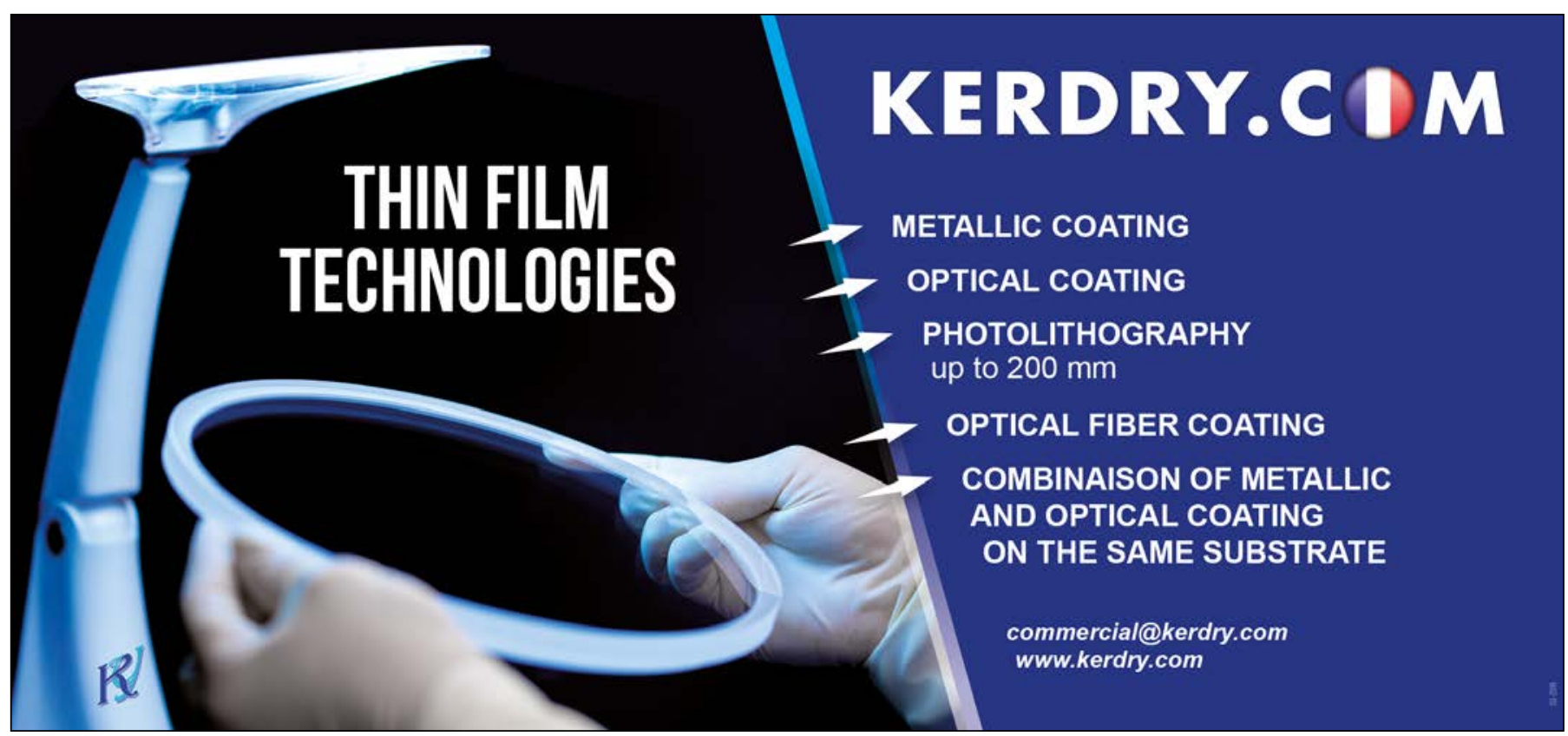




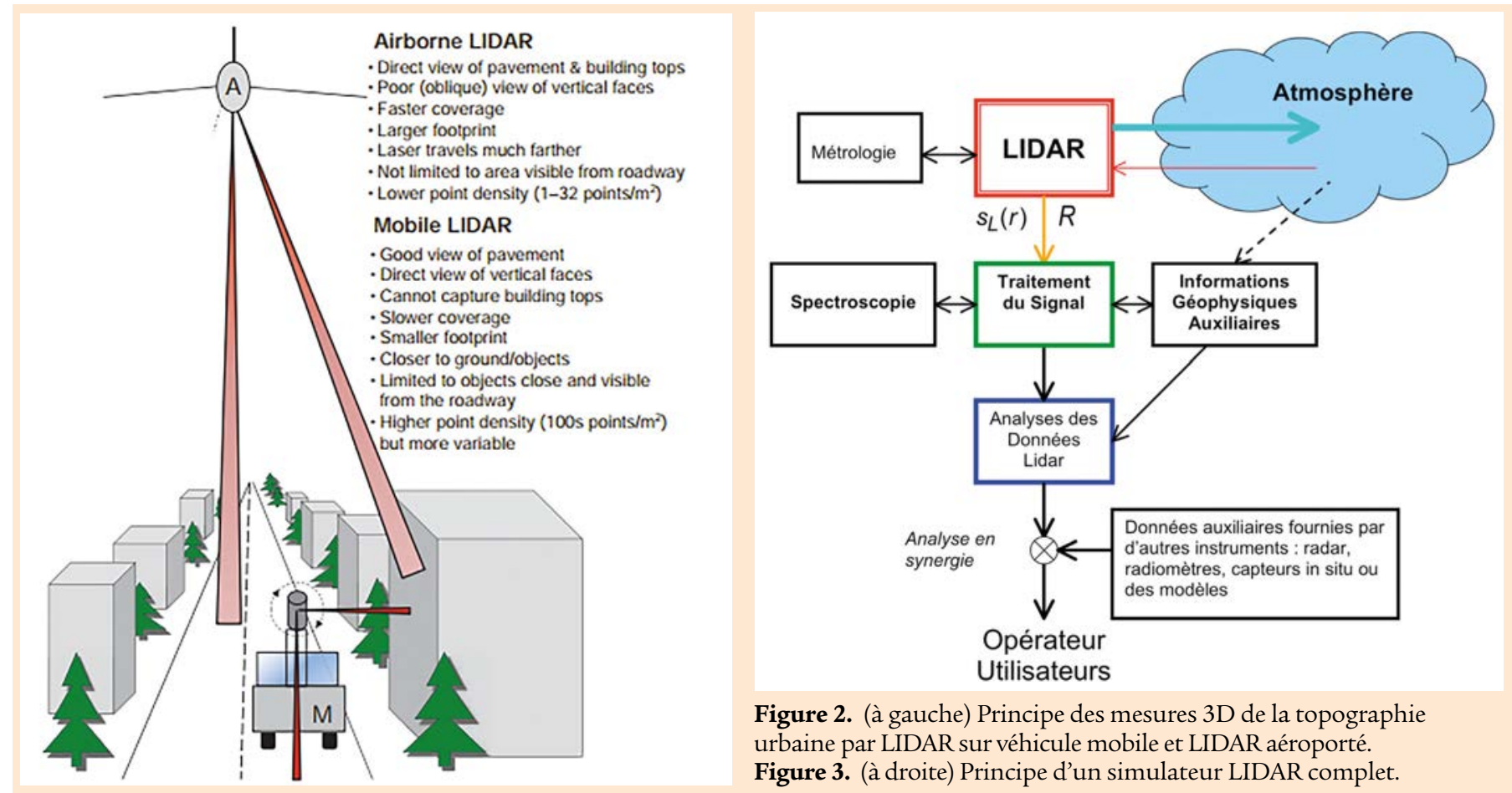

« rétrodiffusion élastique ", le plus simple, (2) LIDAR Raman, (3) LIDAR à absorption différentielle (DiAL pour differential absorption LIDAR), (4) LIDAR à effet Doppler.

Les applications télémétriques utilisent la rétrodiffusion élastique sur des cibles dures. On distingue les LIDARs utilisés à partir du sol (MLS pour mobile LIDAR scanner) ou aéroportés (ALS pour airborne LIDAR scanner) (figure 2). Comme les instruments modernes sont très compacts, on utilise des petits porteurs et des drones. Les applications bathymétriques sont effectuées à partir d'avion ou de bateau.
Pour choisir une méthode LIDAR, on utilise un modèle instrumental de "bout en bout» (end-to-end) comme montré sur la figure 3. La portée LIDAR dépend (1) des constituants du LIDAR (énergie laser, diamètre du télescope, etc.), (2) de l'efficacité du processus de diffusion ou de réflexion des cibles, et (3) de la transmission du milieu (visibilité, turbidité).

\section{Processus d'interaction lumière-matière}

Pour l'atmosphère et les zones aquatiques, les deux processus clé sont : (1) la diffusion et (2) l'atténuation.

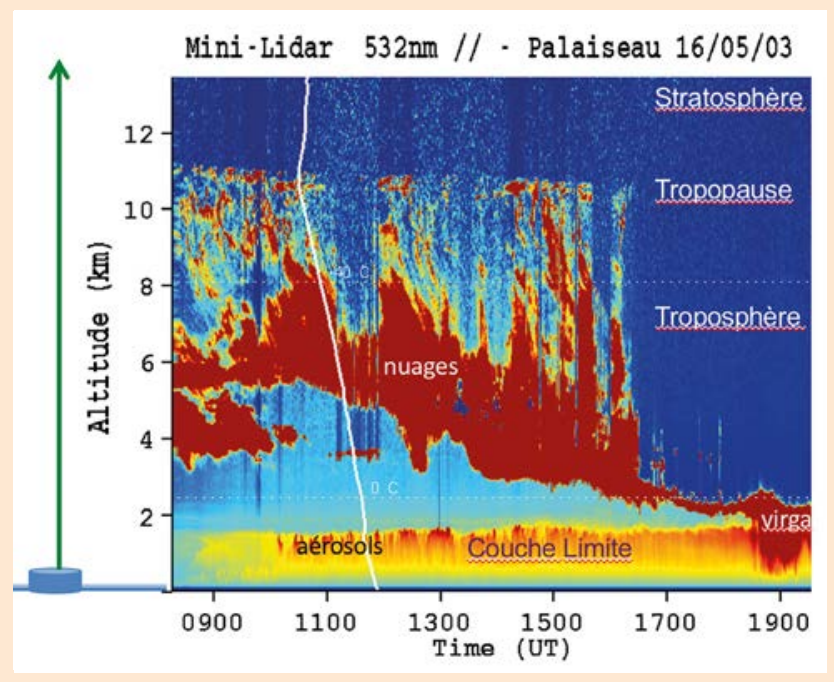

Les caractéristiques de la lumière diffusée peuvent être identiques à celles de la lumière laser ou bien différentes par changement de polarisation, effet Raman, effet Brillouin ou encore effet Doppler. Les cibles dures sont caractérisées par une réflectance spectrale.

La lumière reçue par le télescope se compose de lumière laser rétrodiffusée ou réfléchie additionnée du rayonnement de fond de scène (solaire ou tellurique) à prendre en compte pour le calcul du rapport signal à bruit de la mesure.

\section{Signaux LIDAR}

Pour tous les LIDARs émettant des impulsions laser courtes, la première information pertinente est la distance $r$ entre le LIDAR et la cible. Elle est reliée au temps de vol aller-retour $t$ :

$$
r=\frac{c t}{2}
$$

$c$ étant la vitesse de la lumière dans le milieu (air, eau). La lumière renvoyée est collectée par un télescope solidaire du laser. À ce stade, le signal LIDAR est un bilan optique entre la puissance optique reçue $s_{L}$ (W ou photons par seconde) et l'énergie laser émise ( $E$ en joules ou photons). La relation LIDAR optique est:

$$
\begin{gathered}
s_{L}\left(r, t, \lambda_{L}\right)=K_{L}\left(\lambda_{L}, \lambda_{\ell}\right) g(r) r^{-2} \beta_{\pi}\left(\mathrm{r}, \lambda_{\ell}\right) \\
T_{1}\left(0, r, \lambda_{L}\right) T_{2}\left(r, 0, \lambda_{L}\right)
\end{gathered}
$$


Pour l'atmosphère $\beta_{\pi}\left(r, \lambda_{\ell}\right)$ est le coefficient de rétrodiffusion $\left(\mathrm{m}^{-1} \mathrm{sr}^{-1}\right)$ pour les molécules et les particules dans l'air. $g(r)$ est un facteur géométrique entre 0 à courte distance et $1 . T_{1}\left(0, r, \lambda_{L}\right)$ et $T_{2}\left(r, 0, \lambda_{L}\right)$ sont les transmissions atmosphériques aller et retour. Elles sont identiques pour une diffusion élastique et différentes pour une diffusion Raman. Les transmissions s'écrivent sous une forme exponentielle négative (loi de Beer-Lambert). L'exposant est un coefficient d'extinction $\left(\mathrm{m}^{-1}\right)$.

Le terme instrumental est fonction des composants du LIDAR :

$$
K_{L}\left(\lambda_{L}, \lambda_{\ell}\right)=\frac{c}{2} E A T_{\text {oer }}\left(\lambda_{L}, \lambda_{\ell}\right)
$$

$A$ est la surface du télescope, $T_{\text {orr }}$ la transmission/réflexion des composants optiques. $\lambda_{L}, \lambda_{\ell}$ la longueur d'onde LIDAR après diffusion et la longueur d'onde laser. Les facteurs clé de la mesure sont le produit $E A$ et $\beta_{\pi}\left(r, \lambda_{L}\right)$. Ce dernier peut varier de plusieurs ordres de grandeur. La variation en $\lambda^{-4}$ de la diffusion moléculaire en est une illustration. On écrit les mêmes relations pour la bathymétrie.

La relation (2) ou équation LIDAR suppose des impulsions laser brèves et l'absence de diffusion multiple. Pour restituer les coefficients optiques, on écrit (2) sous une forme simplifiée:

$r^{2} s_{L}(r)=K_{L} \beta_{\pi}(r) \exp \left(-2 \int_{0}^{\mathrm{r}} \alpha(x) d x\right)$

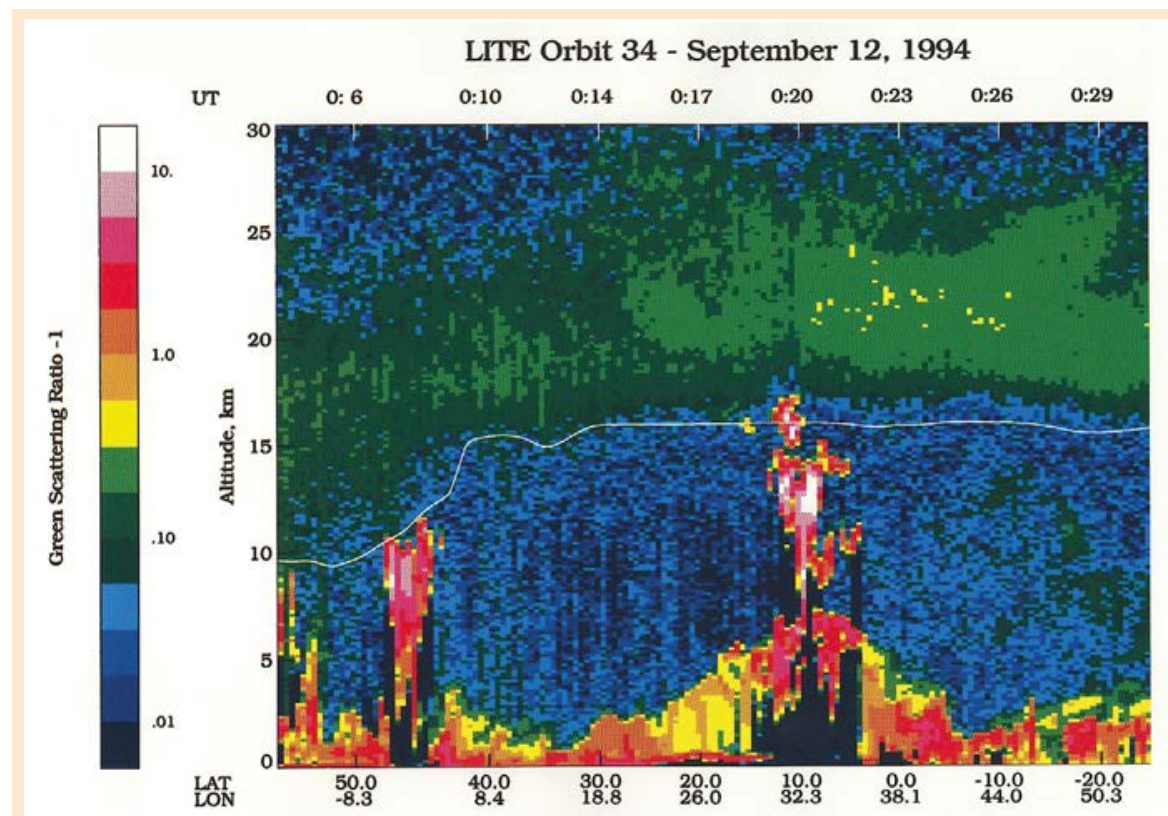

Figure 5. Coupe en latitude/longitude effectuée par le LIDAR spatial LITE/NASA embarqué sur la navette américaine en 1994. Les mesures mettent en évidence les amas nuageux des tropiques et des fronts de latitudes moyennes (rouge et jaune) et les aérosols volcaniques du Mt Pinatubo (en vert). Le trait blanc marque la tropopause thermique. Le code couleur est le rapport de diffusion particulaire.

C'est une relation à deux inconnues ! Pour les particules (aérosols) en suspension dans l'air, on écrit une nouvelle relation entre les coefficients optiques: $\beta_{\pi}(r)=k \alpha(x)$. Avec cette relation, la relation (4) ne contient plus qu'une inconnue $\alpha(x)$. On résout sous la forme d'une équation différentielle de Bernoulli (voir l'article en page 30 de ce numéro) ou d'une équation intégrale.
Pour les composés atmosphériques gazeux, on utilise une méthode différentielle (DiAL). On écrit (4) pour deux longueurs d'onde, l'une absorbée $\left(\lambda_{a}\right)$ par le composé gazeux et l'autre non absorbée $\left(\lambda_{0}\right)$ en référence. On fait le rapport. Les termes identiques s'éliminent:

$$
\frac{s_{L a}(r)}{s_{L o}(r)}=\exp \left(-2 \int_{0}^{\mathrm{r}} \alpha_{a}(x) d x\right)
$$

\section{Filtres Interférentiels}

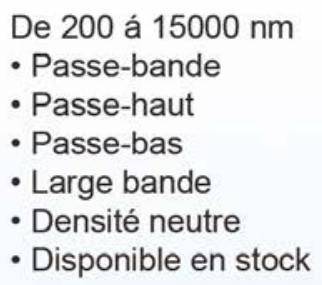

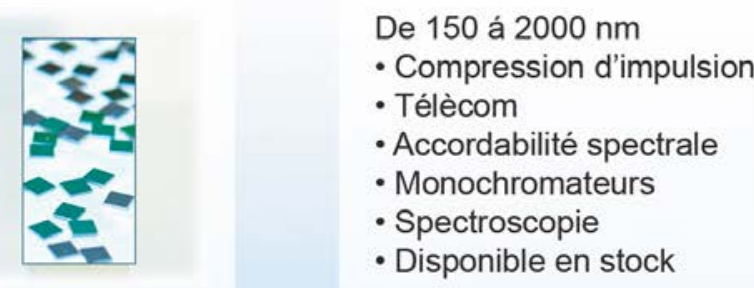

Réseaux Holographiques

UK (parle francais): sales.uk@spectrogon.com·Tel +44 1592770000

Sweden (headquarters): sales.se@spectrogon.com·Tel +46 86382800

US: sales.us@spectrogon.com·Tel+19733311191 


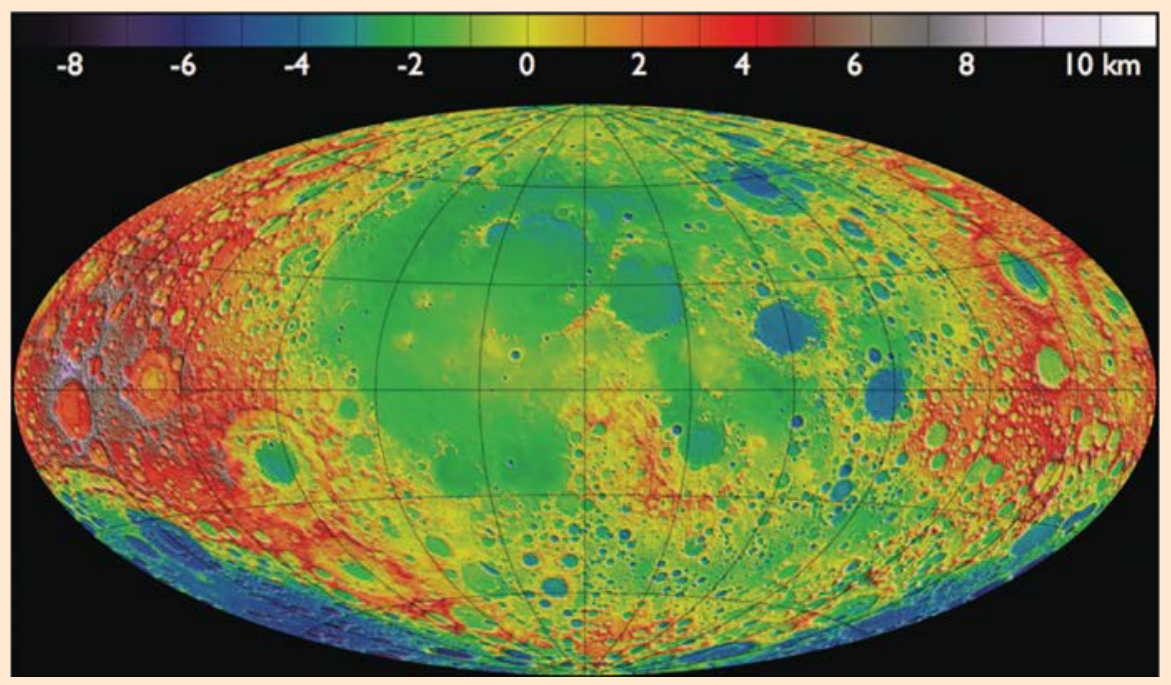

Figure 6. Topographie de la lune établie en 2009 par le LIDAR/NASA LOLA à partir de 1 milliard de mesures. Résolution nord $/ \mathrm{sud}=20 \mathrm{~m}$, résolution est $/$ ouest $=0,1^{\circ}$.

où $\alpha_{a}$ est le coefficient d'absorption. Par choix $\alpha_{0}=0$.

Après calcul :

$$
\alpha_{a}(\mathrm{r})=\frac{1}{2} \frac{d}{d r} \ln \left[\frac{s_{L o}(r)}{s_{L a}(r)}\right]
$$

Connaissant la section efficace d'absorption $\sigma_{a}$ en $\mathrm{m}^{2}$ (donnée spectroscopique), on obtient la concentration : $N_{a}=\frac{\alpha_{a}(r)}{\sigma_{a}}$ en molécules $\mathrm{m}^{-3}$. Les mesures de vitesse utilisent l'effet Doppler (voir l'article en page 36 de ce numéro). On utilise une détection directe ou une détection cohérente suivant l'application.

\section{Les applications}

Les LIDARs permettent d'obtenir d'une part des profils de variables optiques dans l'air et dans l'eau, de concentrations en espèces chimiques minoritaires et de champ de vent, et d'autre part des relevés topographiques et bathymétriques. Suivant les applications, on utilise différents types de plateformes: au sol, bateau, drone, petit ou moyen porteur aéroporté, satellite.

\section{Au sol, aéroporté, drone :}

- Atmosphère : pollution, météorologie, climat, aérosols et nuages, composés minoritaires $\left(\mathrm{H}_{2} \mathrm{O}, \mathrm{O}_{3}, \mathrm{CO}_{2}\right.$, $\mathrm{CH}_{4}$ ), champs de vent. La figure 4 montre un exemple de profil vertical obtenu avec un mini-LIDAR élastique développé pour la recherche.
- Topographie. Végétation : inventaires forestiers, agriculture. Géomorphologie.

- Architecture, archéologie, sismologie et impact météoritique.

- Bathymétrie.

\section{Observation de la Terre :}

- LITE/NASA : 11 jours en 1994, apportant la démonstration que la communauté atmosphérique attendait (figure 5).

-ICESat-1/NASA： 2003-2009. ICESa-2 a fourni des résultats inestimables pour les glaces de mer et continentales, et pour la végétation.

\section{POUR EN SAVOIR PLUS}

\section{LIDARs atmosphériques}

[5] Weitkamp C. Lidar. Springer, Berlin (2005) de l'Ingénieur (2017) de l'Ingénieur (2010)

\section{LIDARs topographiques et bathymétriques} Photogrammetry \& remote Sensing, 54, 164-198 (1999) Photogrammetry \& Remote Sensing, 64, 1-16 (2009) de l'Ingénieur (2010)
- CALIPSO/NASA/CNES : pour l'étude des nuages et des aérosols. Lancé en 2006, toujours en opération. -ADM-Aeolus/ESA : lancé en août 2018 pour la mesure du vent.

- ICESat-2/NASA: lancé en septembre 2018 pour approfondir le suivi des glaces.

\section{Au titre des futures missions :}

- EarthCARE/ESA/JAXA comme la suite de CALIPSO.

- MERLIN/CNES/DLR pour la mesure de la colonne de méthane atmosphérique.

\section{Exploration planétaire:}

- Apollo 15, 16, 17/NASA et les dans les années 70 , pour les premières cartographies de la surface lunaire.

- MOLA/NASA en 1996 pour la cartographie de la planète Mars (figure 6).

La liste n'est pas exhaustive mais l'essentiel est dit.

\section{Conclusion}

Dans cette deuxième décennie du $21^{\mathrm{e}}$ siècle, la technologie LIDAR est partout. La maturité a été atteinte à la fin des années 90 et on peut aujourd'hui parler de plénitude.

[1] Fujii T., Fukuchi T. Laser remote sensing. Taylor \& Francis, Boca Raton, FL, USA (2005)

[2] Hinkley E.D. Laser monitoring of the atmosphere. Springer Verlag, Berlin (1976)

[3] Kovalev V.A., Eichinger W.E. Elastic Lidar. John Wiley \& Sons, New York (2004)

[4] Measures R.M. Laser remote sensing. Krieger, Malabar, FL, USA (1984)

[6] Flamant P.H. Lidars atmosphériques et météorologiques, Principes fondamentaux. Techniques

[7] Flamant P.H., Lidars atmosphériques et météorologiques, méthodes et applications. Techniques

[8] Baltsavias E. Airborne laser scanning: existing systems and firms and other resources. ISPRS Jof

[9] Guenther G., Cunningham A., Larocque P., Reid D. Meeting the accuracy challenge in airborne lidar bathymetry. Proceedings of EARSel SIG Workshop Lidar, Desden/FRG, June 16-17 (2000)

[10] Mallet C., Brétar F. Full-waveform topographic LIDAR: state-of-the-art. ISPRS J of

[11] Shan J., Toth C.K. Topographic laser ranging and scanning. CRC Press, pp. 608, 2008

[12] Flamant P.H. Géolidar pour l'étude des surfaces, de la biosphère et de l'bydrosphère. Techniques 